\title{
Treino Muscular Respiratório Associado à Eletroestimulação Diafragmática em Hemiparéticos
}

\author{
Respiratory Muscle Training Associated with Electrical Stimulation Diaphragmatic in Hemiparesis.
}

\author{
Antonio Gabriel Chagas Queiroz", Daiane Dias da Silva', Rosemary Amorim \\ Correa Lira', Silvia Ramos Fróes Bassini², Edna de Souza Cruz Uematsu ${ }^{3}$
}

\section{RESUMO}

Objetivo. Verificar a efetividade um programa de fortalecimento muscular respiratório associado com eletroestimulação diafragmática transcutânea (EDET) na força respiratória de hemiparéticos. Método. Ensaio clínico randomizado com duração de um mês, no qual participaram 12 indivíduos com hemiparesia decorrente de acidente vascular cerebral. Estes foram divididos em: Grupo A, realizavam um programa de fisioterapia convencional desenvolvido em grupo com duas sessóes semanais de 50 minutos cada; Grupo B, realizavam um programa de fortalecimento muscular respiratório com frequência de 3 sessóes semanais de 30 minutos cada; e Grupo C, realizavam o mesmo programa do Grupo B associado à aplicação de EDET por 10 minutos. Os participantes foram avaliados antes e após o programa de tratamento pela manovacuometria e pela diferença das medidas torácicas e abdominais. Resultados. Houve aumento estatisticamente significante da força inspiratória nos indivíduos do grupo $B(p=0,02)$ e grupo $\mathrm{C}(\mathrm{p}=0,01)$. Conclusáo. Tanto o treino muscular respiratório isolado quanto o mesmo associado com a aplicação de EDET repercutiu em melhora significativa da força inspiratória dos indivíduos deste estudo. O uso do threshold IMT parece ser fundamental para o sucesso do programa de fortalecimento muscular respiratório.

Unitermos. Acidente Vascular Cerebral, Terapia por Exercício, Estimulação Elétrica Nervosa Transcutânea, Diafragma.

Citação. Queiroz AGC, Silva DD, Lira RAC, Bassini SRF, Uematsu ESC. Treino Muscular Respiratório Associado à Eletroestimulação Diafragmática em Hemiparéticos.

\begin{abstract}
Objective. To determine the effectiveness of a program of respiratory muscle strengthening associated with transcutaneous electrical diaphragmatic stimulation (EDET) in respiratory muscle strength in hemiparesis patients. Method. Randomized clinical trial lasting a month, which was attended by 12 individuals with hemiparesis due to a stroke. These were divided into Group A, performed a program conventional physiotherapy developed in group with two weekly sessions of 50 minutes each; Group B, performed a program of muscle strengthening with frequency of 3 weekly sessions of 30 minutes each and Group C performed the same program group B associated with the application of EDET for 10 minutes. The participants were assessed before and after the treatment program by manovacuometry and difference of the measures thoracic and abdominal. Results. There was increase significant statistical of the inspiratory force in the individuals of the group $B(p=0.02)$ and group $C(p=0.01)$. Conclusion. Both the respiratory muscle training isolated how much the same associated with the application of EDET reflected in significant improvement in inspiratory force of individuals in this study. The use of threshold IMT seems to be fundamental to the success of the respiratory muscle strengthening program.
\end{abstract}

Keywords. Stroke, Exercise Therapy, Transcutaneous Electric Nerve Stimulation, Diaphragm.

Citation. Queiroz AGC, Silva DD, Lira RAC, Bassini SRF, Uematsu ESC. CRespiratory Muscle Training Associated with Electrical Stimulation Diaphragmatic in Hemiparesis.
Trabalho realizado na Universidade Cruzeiro do Sul, Sáo Paulo-SP, Brasil.

1. Graduando em Fisioterapia, Universidade Cruzeiro do Sul, Sáo Paulo-SP, Brasil.

2. Fisioterapeuta, Mestre, Docente do curso de Fisioterapia da Universidade Cruzeiro do Sul, São Paulo-SP e Universidade Braz Cubas - UBC, Mogi das Cruzes-SP, Brasil.

3. Fisioterapeuta, Mestre, Docente e supervisora de estágio na área de Fisioterapia Neurofuncional, Universidade Cruzeiro do Sul, São Paulo-SP, Brasil.
Endereço para correspondência: Edna de Souza Cruz Uematsu Clínica de Fisioterapia - Universidade Cruzeiro do Sul Rua Taiuvinha, 31 - Vila Jacuí CEP 08060-040, São Paulo-SP, Brasil. Tel.: (11) 2037-5800.

E-mail: edna.uematsu@cruzeirodosul.edu.br 


\section{INTRODUÇÃO}

O Acidente Vascular Cerebral (AVC) é caracterizado por uma perda local ou global da função encefálica, de início repentino e de origem vascular, com persistência dos sintomas e sinais neurológicos por mais de 24 horas ${ }^{1}$. É a terceira causa de morte no mundo ${ }^{2}$, e quando não leva a óbito gera déficits motores, sensitivos e cognitivos para o indivíduo ${ }^{3-5}$, sendo a fraqueza do hemicorpo contralateral à lesão (hemiparesia) o quadro característico ${ }^{2-4}$.

Essa fraqueza muscular além de atingir os músculos responsáveis pelos movimentos ativos dos membros afeta também os músculos responsáveis pela inspiração (diafragma, escalenos, peitorais maior e menor e intercostais externos) e pela expiração forçada (reto abdominal, oblíquos interno e externo do abdome e transverso do abdome $)^{3,4}$. Dentre os músculos respiratórios afetados destaca-se o diafragma, por ser o principal músculo responsável pelo aumento do diâmetro céfalo-caudal da caixa torácica, permitindo a entrada de ar nos pulmóes (inspiração) $^{1,6-8}$.

Após uma afecção encefálica ocorre uma paralisia da hemicúpula diafragmática do lado do acometimento motor, decorrente de lesóes centrais no trajeto das vias córtico-diafragmáticas ${ }^{9}$. Foi observada diminuição da força respiratória e da mobilidade tóraco-abdominal em hemiparéticos crônicos quando comparados a um grupo controle de indivíduos saudáveis ${ }^{10}$. Além disso, devido à fraqueza dos músculos abdominais, ocorre uma elevação do gradil costal e a caixa torácica se apresenta em uma posição inspiratória, interferindo, assim, na ação do músculo diafragma ${ }^{11}$. Dessa forma a mecânica respiratória é modificada, já que os músculos abdominais proporcionam estabilidade de tronco para que os músculos respiratórios hajam de maneira eficiente $e^{4,11}$.

Essa alteração na mecânica respiratória normal faz com que o indivíduo entre em fadiga rapidamente, comprometendo seu desempenho na realização de suas atividades de vida diária ${ }^{3,4}$.

Para treinar o músculo diafragma vários recursos são utilizados, dentre os quais se destacam a eletroestimulação transcutânea e o fortalecimento muscular ${ }^{3,8,11,12}$. A eletroestimulação diafragmática transcutânea (EDET) consiste em gerar, através de eletrodos colocados em pontos motores, potenciais de ação no nervo frênico que estimulem as fibras diafragmáticas a contrair de forma mais eficaz ${ }^{8,13}$. Foi observado aumento de força respiratória utilizando um protocolo de EDET em um indivíduo saudável ${ }^{12}$.

Já o fortalecimento muscular tem como princípio submeter o músculo a uma carga além da habitual com o objetivo de tornar sua contração mais potente ${ }^{7,13}$. Há relato de resultados positivos com relação à força respiratória após um programa de fortalecimento muscular associado com treinamento cardiovascular em indivíduos com hemiparesia decorrente de $\mathrm{AVC}^{3}$.

Diante das repercussóes respiratórias geradas pela hemiparesia, propõe-se um treino muscular respiratório associando a EDET em pacientes hemiparéticos, com o objetivo de verificar a efetividade desse programa na força respiratória desses indivíduos.

\section{MÉTODO}

\section{Amostra}

Trata-se de um ensaio clínico randomizado de alocação aleatória desenvolvido na Clínica-escola da Universidade Cruzeiro do Sul, no qual participaram 12 pacientes, de ambos os sexos, com idade entre 30 e 70 anos e com hemiparesia decorrente de AVC.

Foram incluídos no estudo indivíduos com hemiparesia pós-AVC com mais de seis meses de acontecimento do evento neurológico, com funçôes cognitivas suficientes para atenderem aos comandos e com liberação médica para a intervenção.

Foram adotados como critérios de exclusão: pacientes com doenças coronarianas e cardiovasculares graves; com doenças respiratórias; fumantes; e com alteraçôes osteoarticulares que restrinjam a amplitude de movimento.

Este estudo foi aprovado pelo Comitê de Ética em Pesquisa da Universidade Cruzeiro do Sul, protocolo número 339/082012 e os indivíduos assinaram um termo de consentimento livre e esclarecido, conforme determina a resolução 196/96 do Conselho Nacional de Saúde.

\section{Procedimentos}

Os quatro participantes do grupo convencional 
(grupo A) faziam parte de um programa de fisioterapia sem enfoque para a musculatura respiratória (alongamentos globais, fortalecimentos globais e treinos de equilíbrio e marcha) oferecido pela Clínica-escola da Universidade Cruzeiro do Sul. Os demais participantes foram divididos aleatoriamente e proporcionalmente em dois grupos distintos (grupo B e grupo C) através de sorteio. O sorteio foi realizado seguindo as seguintes etapas: um dos autores do estudo numerou os indivíduos de 1 a 8; após isso papéis com a mesma numeração foram depositados em urna; por fim, um indivíduo que não fazia parte do estudo retirava um papel por vez da urna, sendo os sujeitos alocados 1:1 em grupo B e grupo C, respectivamente.

No grupo B (fortalecimento respiratório) os pacientes foram submetidos ao treino de fortalecimento da musculatura respiratória iniciando com alongamentos de escalenos, esternocleidomastoideos, peitorais, trapézios, rotadores internos de ombro e flexores de punho seguido de exercícios respiratórios associados com flexão de ombro à $90^{\circ}$ e com os padróes respiratórios com tempo inspiratório e expiratório 1:1 e 2:1, exercícios diafragmáticos com resistência elástica, exercícios utilizando um incentivador respiratório (threshold) e finalizando com relaxamento (exercícios de respiração profunda).

No grupo C (fortalecimento respiratório associado à EDET) os pacientes além de realizarem o mesmo protocolo do grupo B foram também submetidos à aplicação de EDET sendo colocado um eletrodo ao nível da $4^{\text {a }}$ vértebra cervical (raiz nervosa do nervo frênico) e outro logo abaixo do processo xifóide do esterno (ventre muscular do músculo diafragma)8. Os parâmetros utilizados foram: freqüência de $50 \mathrm{~Hz}$, largura de pulso de 200 microssegundos, tempo de 10 minutos e intensidade no limiar motor com tetanização ${ }^{14}$.

Todos os exercícios foram realizados inicialmente em três séries de 10 repetiçóes com o paciente na posição de semi-flower, sendo reprogramadas as séries e a carga de acordo com a evolução do tratamento.

Foram utilizados aparelhos de eletroestimulação transcutânea da marca Quark modelo Fes 991, eletrodos, bastôes, caneleiras de 0,5 e $1 \mathrm{~kg}$, faixas elásticas e threshold.

O programa do grupo intervenção (grupo $\mathrm{B}$ e grupo C) teve três sessóes semanais de 30 a 40 minutos cada. Já o grupo controle (grupo A) fazia parte de um programa de fisioterapia convencional desenvolvido em grupo com frequência de duas sessôes semanais de $50 \mathrm{mi}$ nutos cada. Os três programas tiveram duração de um mês.

Todos os participantes passaram por duas avaliaçôes, sendo uma ao início do programa e outra no término do tratamento. As avaliaçôes e as intervençôes foram realizadas por três autores do estudo previamente treinados em relação aos procedimentos.

Foram medidas a força expiratória (PEmáx) e inspiratória (PImáx) através de um manovacuômetro da marca Globalmed modelo MVD 300, no qual os pacientes realizaram a medida da PImáx partindo da capacidade residual funcional até a capacidade inspiratória máxima, e a PEmáx partindo da capacidade inspiratória máxima até a capacidade residual funcional ${ }^{15}$ (os indivíduos realizavam as inspiraçóes e expiraçóes pela cavidade oral em um bocal conectado ao aparelho). Foram adotados como referência os valores de normalidade da PImáx e da PEmáx em um adulto jovem, que são, respectivamente, entre -90 a $-120 \mathrm{cmH} 2 \mathrm{O}$ e entre 100 a $150 \mathrm{cmH} 2 \mathrm{O}$, de modo que a partir dos 20 anos de idade esse valor decresce anualmente em $0,5 \mathrm{cmH} 2 \mathrm{O}^{13}$.

As medidas torácicas e abdominais foram realizadas com os participantes na posição de decúbito dorsal durante uma inspiração e uma expiração, e o avaliador com uma fita métrica mediu o diâmetro torácico (ao nível do processo xifóide do osso esterno) e abdominal (ao nível da cicatriz onfálica ${ }^{16}$.

Os valores de PEmáx e PImáx serviram de referência para a utilização do Threshold $\mathrm{PEP}$ (pressão positiva na expiração) e IMT (treino muscular inspiratório), respectivamente. Este incentivador é formado por uma estrutura externa cilíndrica de material plástico, e uma válvula acoplada a uma mola que proporciona uma resistência constante e específica.

Náo houve diferença no recrutamento muscular utilizando $20 \%$, $30 \%$ ou $40 \%$ do valor de referência da manovacuometria ${ }^{17}$. Dessa forma, os autores do presente estudo ajustaram o threshold com $30 \%$ dos valores de referência da manovacuometria, já que os mesmos julgam este como um valor seguro. Os exercícios com esse incentivador foram realizados em três séries de 5 repetições 
cada, de modo que quando este valor de referência não era possível de ser ajustado, colocava-se o valor mínimo do aparelho.

\section{Análise Estatística}

As variáveis avaliadas foram expressas através de média e desvio padrão e o ganho pós-intervenção através de porcentagem. Para a comparação avaliação x reavaliação foi utilizado o teste t de Student pareado, adotando nível de significância de $5 \%(\mathrm{p} \leq 0,05)$.

\section{RESULTADOS}

A Tabela 1 mostra as variáveis demográficas dos indivíduos que compóem o estudo. Com relação ao gênero, a amostra foi composta por 7 homens $(58,33 \%)$ e 5 mulheres $(41,66 \%)$.

A média geral de idade dos indivíduos foi de $54,08 \pm 11,37$ anos, com o tempo médio de acometimento da doença de $65,75 \pm 56,59$ meses, sendo o tipo isquêmico mais comum $(66,66 \%)$.

Levando-se em conta o acometimento do lado do corpo, a amostra foi composta por 10 indivíduos ta foi

Tabela 1.

Descrição das características demográficas dos indivíduos do estudo.

\begin{tabular}{lcccc}
\hline & Grupo A & Grupo B & Grupo C & Total \\
\hline $\begin{array}{l}\text { Número de } \\
\text { indivíduos }\end{array}$ & 4 & 4 & 4 & 12 \\
\hline $\begin{array}{l}\text { Gênero (\%) } \\
\text { Masculino }\end{array}$ & 50 & 75 & 50 & 58,33 \\
Feminino & 50 & 25 & 50 & 41,66 \\
\hline $\begin{array}{l}\text { Tipo de AVC } \\
\text { (\%) }\end{array}$ & & & & \\
$\begin{array}{l}\text { Isquêmico } \\
\text { Hemorrágico }\end{array}$ & 25 & 25 & 50 & 63,33 \\
\hline $\begin{array}{l}\text { Hemicorpo } \\
\text { afetado (\%) }\end{array}$ & 100 & 100 & 50 & 83,66 \\
$\begin{array}{l}\text { Direito } \\
\text { Esquerdo }\end{array}$ & 0 & 0 & 50 & 16,33 \\
\hline $\begin{array}{l}\text { Idade média } \\
\text { (meses) }\end{array}$ & $52,5 \pm 13,37$ & $52,75 \pm 15,19$ & $57 \pm 6,87$ & $54,08 \pm 11,37$ \\
\hline $\begin{array}{l}\text { Tempo } \\
\text { médio de } \\
\text { ocorrência } \\
\text { do AVC } \\
\text { (meses) }\end{array}$ & $58,5 \pm 34,69$ & $84 \pm 84,07$ & $54,75 \pm 52,89$ & $65,75 \pm 56,59$ \\
\hline
\end{tabular}

Média \pm desvio padrão (DP), AVC: Acidente Vascular Cerebral hemiparesia a direita $(83,66 \%)$ e 2 sujeitos com o hemicorpo esquerdo afetado (16,33\%).

A Tabela 2 mostra que no grupo A, a média de PImáx inicial foi de $-15 \mathrm{cmH} 2 \mathrm{O}$ e a final foi de $-17,5$ $\mathrm{cmH} 2 \mathrm{O}$ (aumento de 16,66\%), enquanto que no grupo $\mathrm{B}$ a média inicial foi de $-22,75 \mathrm{cmH} 2 \mathrm{O}$ e a final foi de $-31,5$ cmH2O (aumento de 38,46\%), e no Grupo C a PImáx inicial foi de $-13,5 \mathrm{cmH} 2 \mathrm{O}$ e a final de $-26,5$ cmH2O (aumento de 96,29\%). Houve diferença pós-intervenção no grupo $\mathrm{B}(\mathrm{p}=0,02)$ e grupo $\mathrm{C}(\mathrm{p}=0,01)$.

Já com relação aos valores de PEmáx (Tabela 3), as médias inicias e finais obtidas foram, respectivamente, de $25,25 \mathrm{cmH} 2 \mathrm{O}$ e 23,25 cmH2O no Grupo A (diminuição de 7,92\%), de $26,75 \mathrm{cmH} 2 \mathrm{O}$ e $33,75 \mathrm{cmH} 2 \mathrm{O}$ no Grupo B (aumento de 26,16\%), e 22 cmH2O e 35,25 cmH2O no Grupo C (aumento de 60,22\%).

$\mathrm{Na}$ diferença das medidas torácicas (Tabela 4), os valores médios iniciais e finais obtidos foram, respectivamente, $1 \mathrm{~cm}$ e $1 \mathrm{~cm}$ no grupo A (manutenção dos valores), $1,375 \mathrm{~cm}$ e $2 \mathrm{~cm}$ no grupo B (aumento de 45,45\%), e 1,375 e 1,125 cm no grupo C (diminuição de 14,54\%).

Por fim, a Tabela 5 aponta que no grupo A, a média da diferença das medidas abdominais inicial foi

Tabela 2.

Média ( \pm desvio padrão) dos valores de pressão inspiratória máxima (PImáx em cmH2O) pré e pós-intervenção dos grupos.

\begin{tabular}{lcccc}
\hline & PImáx inicial & PImáx final & $\begin{array}{c}\text { Diferença } \\
\text { pré e pós }\end{array}$ & $\mathbf{p}$ \\
\hline Grupo A & $-15 \pm 11,91$ & $-17,5 \pm 11,09$ & $16,66 \%$ & 0,28 \\
Grupo B & $-22,75 \pm 10,07$ & $-31,5 \pm 12,76$ & $38,46 \%$ & 0,02 \\
Grupo C & $-13,5 \pm 10,72$ & $-26,5 \pm 12,34$ & $96,29 \%$ & 0,01 \\
\hline
\end{tabular}

Tabela 3.

Média ( \pm desvio padrão) dos valores de expiratória máxima (PEmáx em cmH2O) pré e pós-intervenção dos grupos.

\begin{tabular}{lcccc}
\hline & PEmáx inicial & PEmáx final & $\begin{array}{c}\text { Diferença } \\
\text { pré e pós }\end{array}$ & p \\
\hline Grupo A & $25,25 \pm 14,17$ & $23,25 \pm 10,56$ & $-7,92 \%$ & 0,60 \\
Grupo B & $26,75 \pm 22,89$ & $33,75 \pm 20,13$ & $26,16 \%$ & 0,21 \\
Grupo C & $22 \pm 18,11$ & $35,25 \pm 24,24$ & $60,22 \%$ & 0,11 \\
\hline
\end{tabular}


Tabela 4.

Valores médios ( \pm desvio padrão) da diferença das medidas torácicas (MT em $\mathrm{cm}$ ) antes e depois da intervenção.

\begin{tabular}{ccccc}
\hline & MT inicial & MT final & $\begin{array}{c}\text { Diferença } \\
\text { pré e pós }\end{array}$ & p \\
\hline Grupo A & $1 \pm 1$ & $1 \pm 0,4$ & $0 \%$ & 1 \\
Grupo B & $1,375 \pm 1,1$ & $2 \pm 1,41$ & $45,45 \%$ & 0,07 \\
Grupo C & $1,375 \pm 0,75$ & $1,125 \pm 0,62$ & $-14,54 \%$ & 0,39 \\
\hline
\end{tabular}

de $1 \mathrm{~cm}$ e a final de $2 \mathrm{~cm}$ (aumento de 100\%), enquanto que no grupo $\mathrm{B}$ a média inicial foi de $1,875 \mathrm{~cm}$ e a final de $4 \mathrm{~cm}$ (aumento de 113,33\%), e no grupo C o valor da diferença entre as medidas abdominais inicial foi de $1,125 \mathrm{~cm}$ e a final de $2,125 \mathrm{~cm}$ (aumento de $88,88 \%$ ).

\section{DISCUSSÃO}

Os resultados desse estudo mostram que com relação à PImáx, no grupo $\mathrm{B}$, houve um acréscimo de $38,46 \%$ e no grupo C de $96,29 \%$. O threshold foi utilizado nos grupos $\mathrm{B}$ e $\mathrm{C}$, que obtiveram maior ganho de força respiratória quando comparados ao grupo controle. Um estudo realizou um programa de treino muscular inspiratório usando o threshold por 30 minutos, 7 vezes por semana durante 12 semanas e verificaram um incremento de $115 \%$ na PImáx de indivíduos com insuficiência cardíaca $^{18}$. Já outros autores usaram um protocolo de fortalecimento muscular inspiratório com o threshold em hemiparéticos por 30 minutos, 5 vezes por semana durante 8 semanas que resultou em melhora da força inspiratória verificada pela manovacuometria ${ }^{19}$. Semelhante a isso, houve um aumento da PImáx e PEmáx em pacientes portadores de esclerose múltipla utilizando um treino da musculatura inspiratória com threshold em dias alternados, com 3 séries de 10 repetiçóes por um período de 10 semanas ${ }^{20}$.

Os resultados ainda apontam que a porcentagem de ganho de força inspiratória se mostrou maior nos indivíduos que realizaram a EDET (grupo C). Em um estudo utilizando um protocolo de EDET em ratos por 5 dias foi verificado melhora mais efetiva das condiçôes metabólicas dos músculos respiratórios dos ratos que utilizaram a EDET quando comparados aos do grupo controle ${ }^{21}$. Outro estudo usou diferentes protocolos com 12 sessões de aplicação de EDET em dois grupos de mulheres sau-
Tabela 5.

Valores médios ( \pm desvio padräo) da diferença das medidas abdominais (MA em cm) pré e pós-intervenção.

\begin{tabular}{ccccc}
\hline & MA inicial & MA final & $\begin{array}{c}\text { Diferença } \\
\text { pré e pós }\end{array}$ & p \\
\hline Grupo A & $1 \pm 0,4$ & $2 \pm 1,08$ & $100 \%$ & 0,09 \\
Grupo B & $1,875 \pm 1,54$ & $4 \pm 1,22$ & $113,33 \%$ & 0,09 \\
Grupo C & $1,125 \pm 0,62$ & $2,125 \pm 1,79$ & $88,88 \%$ & 0,32 \\
\hline
\end{tabular}

dáveis e verificaram que ambos promoveram aumento da força inspiratória e expiratória, diferentemente do grupo controle que não alterou os seus resultados ${ }^{22}$. Alguns autores propóem que o tempo mínimo de aplicação da EDET deve ser de 20 minutos $^{14,21}$. Contudo, o tempo utilizado nesse estudo foi de 10 minutos já que tempos maiores poderiam predispor os indivíduos à fadiga, visto que os mesmos já haviam realizado fortalecimento específico para a musculatura respiratória antes da aplicação. Quanto às medidas torácicas pós-tratamento, houve no grupo A manutenção dos valores, no grupo B houve aumento de $45,45 \%$ e já no grupo C houve diminuição de $14,54 \%$. Quanto aos valores das medidas abdominais, o grupo A obteve aumento de $100 \%$, o grupo B de $113,33 \%$ e o grupo C de $88,88 \%$. Náo houve diferença significante em nenhum dos grupos.

O grupo $\mathrm{C}$ pode não ter alcançado melhores valores nessas medidas porque os indivíduos desse grupo apresentavam uma idade média mais avançada quando comparados aos sujeitos do grupo A e B. Os valores normais de mobilidade tóraco-abdominal é no mínimo $5 \mathrm{~cm}$ e esses valores começam a diminuir com o avanço da ida$\mathrm{de}^{13}$. Outros fatores que podem ter influenciado nestes resultados foram a assiduidade dos indivíduos, o número limitado de sessóes e a falta de enfoque para exercícios que visam o aumento da mobilidade torácica e abdominal.

\section{CONCLUSÕES}

Os achados apontam que tanto o treino muscular respiratório isolado quanto o mesmo associado com a aplicação de EDET repercutiu em melhora da força inspiratória dos indivíduos com hemiparesia decorrente de um AVC deste estudo. O uso do threshold IMT parece ser fundamental para o sucesso do programa de fortale- 
cimento muscular respiratório. Por fim, diante dos resultados, sugere-se que treino muscular respiratório e a EDET não tem influência sobre a mobilidade torácica e abdominal. Para a confirmação dos achados encontrados nesse estudo, fazem-se necessárias novas pesquisas com tamanho amostral maior e mais homogêneo, além de um período de intervenção mais longo.

\section{REFERÊNCIAS}

1. Glória AE, Nascimento LR, Paiva CMR, Fernandes MS, Lima RCM, Moura RMF. Treinamento global na pressão inspiratória máxima e funcionalidade de um indivíduo com hemiparesia crônica. Consci Saúde 2011;10:555-62.

2.Soares AV, Santos SBO, Ferreira PR, Schueroff B, Woellner SS, Domenech $\mathrm{SC}$ et al. Cicloergometria adaptada para pacientes hemiparéticos pós-acidente vascular encefálico. Neurociências 2011;7:18-24.

3.Castro JB, Abilel JC, Lavísio EM, Uematsu ESC, Moraes JV, Silva A. Treinamento em Esteira e Fortalecimento Muscular no Tratamento de Hemiparéticos Crônicos. Rev Neurocienc 2011;19:423-32.

4.Pompeu SMAA, Pompeu JE, Rosa M, Silva MR. Correlação entre função motora, equilíbrio e força respiratória pós um Acidente Vascular Cerebral. Rev Neurocienc 2011;19:614-20.

5.Carvalho FA. Além da incapacidade motora: uma visão sobre outra seqüela pouco falada do AVC. Rev Neurocienc 2011;19:14-5.

6.West JB. Fisiologia Respiratória. 6. ed. São Paulo: Manole, 2002, 199p.

7.Costa D. Fisioterapia Respiratória Básica. São Paulo: Atheneu, 2004, 127p.

8.Silva EG, Vieira D. Estimulação Diafragmática Elétrica Transcutânea na melhora do metabolismo da musculatura respiratória: revisão. Rev Min Ciênc Saúde - UNIPAM 2009;1: 68-80.

9.Kumar S, Reddy R, Prabhakar S. Contralateral diaphragmatic palsy in acute stroke: an interesting observation. Indian J Crit Care Med 2009;13:28-30. http://dx.doi.org/10.4103/0972-5229.531132

10.Teixeira-Salmela LF, Parreira VF, Britto RR, Brant TC, Inácio EP, Alcântara TO, et al. Respiratory pressures and thoracoabdominal motion in community-dwelling chronic stroke survivors. Arch Phys Med Rehabil 2005;86:1974-8. http://dx.doi.org/10.1016/j.apmr.2005.03.035
11.Davies PM. Exatamente no Centro. Atividade seletiva do tronco no tratamento da hemiplegia no adulto. Barueri: Editora Manole, 1996, 284p.

12.Barros JL, Capeletti AM. Alteraçôes da ventilação voluntária máxima através da estimulação diafragmática elétrica transcutânea em um indivíduo saudável. Um estudo de caso. Rev eletr Saúde Pesq Refl 2011;1:1-14.

13.Azeredo CAC. Fisioterapia Respiratória Moderna. 4a ed. São Paulo: Manole, 2002, 505p.

14.Cunha CS, Santana ERM, Fortes RA. Técnicas de fortalecimento da musculatura respiratória auxiliando o desmame do paciente em ventilaçáo mecânica invasiva. Cad UniFOA 2008;6:80-6.

15.Silva FC, Silva SM, Sampaio LMM, Corrêa JCF, Corrêa FI. Relação entre recuperação motora e força muscular respiratória de hemiparéticos crônicos e agudos após acidente vascular encefálico. Ter Man 2012;10:1:6.

16.Caldeira VS, Starling CCD, Britto RR, Martins JA, Sampaio RF, Parreira VF. Precisão e acurácia da cirtometria em adultos saudáveis. J Bras Pneumol 2007;33:519-26. http://dx.doi.org/10.1590/S1806-37132007000500006

17.Souza E, Terra ELSV, Pereira R, Chicayban L, Silva J, Sampaio-Jorge F. Análise eletromiográfica do treinamento muscular inspiratório sob diferentes cargas do threshold ${ }^{\circledR}$ imt. Rev Perspe 2008;2:103-12.

18.Dall'Ago P, Chiappa GRS, Guths H, Stein R, Ribeiro JP. Inspiratory Muscle Training in Patients With Heart Failure and Inspiratory Muscle Weakness. J Am Col Cardiol 2006;47:757-63. http://dx.doi.org/10.1016/j. jacc.2005.09.052

19.Britto RR, Rezende NR, Marinho KC, Torres JL, Parreira VF, Teixeira-Salmela LF. Inspiratory muscular training in chronic stroke survivors: a randomized controlled trial. Arch Phys Med Rehabil 2011;92:184-90. http://dx.doi. org/10.1016/j.apmr.2010.09.029

20.Klefbeck B, Hamrah Nedja J. Effect of inspiratory muscle training in patients with multiple sclerosis. Arch Phys Med Rehabil 2003;84:994-9. http:// dx.doi.org/10.1016/S0003-9993(03)00133-3

21. Cancelliero KM, Costa D, Silva CA. Estimulação diafragmática elétrica transcutânea melhora as condições metabólicas dos músculos respiratórios de ratos. Rev Bras Fisioter 2006;10:59-65. http://dx.doi.org/10.1590/S141335552006000100008

22.Cancelliero KM, Ike D, Sampaio LMM, Santos VLA, Stirbulov R, Costa D. Estimulação diafragmática elétrica transcutânea (EDET) para fortalecimento muscular respiratório: estudo clínico controlado e randomizado. Fisioter Pesq 2012;19:303-8. http://dx.doi.org/10.1590/S1809-29502012000400002 